\title{
MEP monitoring during aortic surgery: what we truly know
}

\author{
N. A. Sutedja ${ }^{1}$ A. W. H. Hollands ${ }^{1}$ M. J. Jacobs ${ }^{2,3}$
}

Received: 21 March 2017 / Accepted: 18 April 2017 / Published online: 2 May 2017

(C) Japanese Society of Anesthesiologists 2017

\section{To the Editor:}

We read with interest the meta-analysis by Tanaka et al. [1]. Indeed MEP monitoring is an excellent tool for assessing spinal ischemia. However, we believe that the authors' conclusions should be discussed even more thoroughly.

We were alerted by their summary of data published by our group as 16 patients were classified as 'false positive'. What may have caused a misinterpretation, were the cases in which the MEP changes were transient. After intervention, the MEPs fully recovered; consequently, these patients had no paraparesis. In our opinion these cases should be regarded as 'true negative'. After re-analysis of all publications, we were able to re-categorize several test results.

However, some false positives and negatives still remained. False positives might be explained by differences in methodology and interpretation of MEP monitoring. No information was provided on the minimal amplitude of the MEPs or how differentiation between central and peripheral ischemia was made. The two remaining false negatives after our re-analysis could represent delayed ischemia,

Electronic supplementary material The online version of this article (doi:10.1007/s00540-017-2367-6) contains supplementary material, which is available to authorized users.

This comment refers to the article available at doi:10.1007/s00540-016-2242-x.

A. W. H. Hollands

a.hollands@student.maastrichtuniversity.nl

1 Department of Clinical Neurophysiology, Maastricht University Medical Center, Maastricht, The Netherlands

2 Department of Vascular Surgery, Maastricht University Medical Center, Maastricht, The Netherlands

3 University Hospital Aachen, Aachen, Germany occurring after surgery. This is supported by the reported postoperative low perfusion pressures in both cases.

In conclusion, the sensitivity and specificity of MEP monitoring to detect spinal ischemia is even better. Due to the importance of sound methodology and unambiguous interpretation of MEPs, a uniform approach to MEP monitoring should be realized. This should include recommendations for anesthesia and stimulation regimens as well as clearly defining outcome measures. We propose that a consensus guideline should be generated by key players with experience in MEP monitoring.

\section{Reference}

1. Tanaka Y, Kawaguchi M, Noguchi Y, Yoshitani K, Kawamata M, Masui K, Nakayama T, Yamada Y. Systematic review of motor evoked potentials monitoring during thoracic and thoracoabdominal aortic aneurysm open repair surgery: a diagnostic metaanalysis. J Anesth. 2016;30:1037-50. 